\title{
Preoperative Factors Associated with Infiltrative Histologic Growth Patterns in Extremity Soft Tissue Sarcoma
}

\author{
Jong Woong Park, ${ }^{1}$ Han-Soo Kim, ${ }^{1}$ Cheol Lee, ${ }^{2}$ Hye Jin Yoo, ${ }^{3}$ Ji Yeon Yun, ${ }^{1}$ and Ilkyu Han \\ ${ }^{1}$ Department of Orthopaedic Surgery, Seoul National University Hospital, Seoul, Republic of Korea \\ ${ }^{2}$ Department of Pathology, Seoul National University College of Medicine, Seoul, Republic of Korea \\ ${ }^{3}$ Department of Radiology, Seoul National University Hospital, Seoul, Republic of Korea \\ Correspondence should be addressed to Ilkyu Han; hik19@snu.ac.kr
}

Received 1 February 2017; Revised 6 May 2017; Accepted 19 June 2017; Published 20 July 2017

Academic Editor: R. Lor Randall

Copyright (c) 2017 Jong Woong Park et al. This is an open access article distributed under the Creative Commons Attribution License, which permits unrestricted use, distribution, and reproduction in any medium, provided the original work is properly cited.

\begin{abstract}
Soft tissue sarcoma (STS) with an infiltrative histologic growth pattern, when compared to STS with an expansile pattern, may pose difficulties in local control. Preoperative assessment of the presence of infiltrative histologic growth pattern would be helpful in deciding treatment strategies. A review of 144 patients who underwent surgery for extremity STS was performed. Microscopically, the histologic growth pattern was defined as infiltrative if the penetration of the tumor cells into the surrounding tissue was observed. Possible clinicopathologic factors that might be associated with infiltrative histologic growth pattern were investigated with regard to patient demographics, tumor characteristics, and MRI findings. Of the 144 tumors, 71 (49\%) showed infiltrative histologic growth pattern. On multivariate analysis, histological subtypes other than liposarcoma $(\mathrm{OR}=4.57, p=0.02)$ and infiltrative border on MRI (OR $=2.48, p=0.01)$ were independent factors associated with infiltrative histologic growth pattern. Predictive index based on these two factors showed a significant improved accuracy (ROC-AUC $=0.647$ ) for predicting infiltrative histologic growth pattern compared to either factor alone. Our data suggests that liposarcoma histology and tumor border on MRI can predict histologic growth pattern in extremity STS.
\end{abstract}

\section{Introduction}

Local recurrence in extremity soft tissue sarcomas (STS) not only is associated with poor oncologic outcome but also has deleterious effects on limb function [1-4]. Local recurrence is largely dictated by the ability to achieve histologically negative resection margins, and failure to obtain histologically negative margins represents microscopic residual disease and translates into high rates of local recurrence $[5,6]$. To obtain histologically negative resection margin, accurate determination of histologic tumor extent is necessary.

Histologic growth pattern has been associated with local recurrence in extremity STS [7-9]. STS with an infiltrative histologic growth pattern, when compared to STS with an expansile growth pattern, may pose difficulties in local control and subsequent risk for local recurrence $[10,11]$. Thus, preoperative assessment of the infiltrative histologic growth pattern, using preoperatively available clinicopathologic parameters, would be helpful in guiding surgery and adjuvant treatment. The purpose of this study was to identify clinicopathologic characteristics that are predictive of infiltrative histologic growth pattern in extremity STS.

\section{Material and Methods}

2.1. Patient Selection. From our institutional database, 289 patients who underwent curative surgery for extremity STS between 2009 and 2014 were retrospectively reviewed. For the purpose of analysis, 109 patients in whom histologic growth pattern of the entire tumor periphery could not be evaluated were excluded. Of the remaining 180 patients, patients with well-differentiated liposarcoma $(n=24)$, patients for whom histological grade could not be evaluated $(n=10)$, and patients without MRI $(n=2)$ were also excluded, which left 


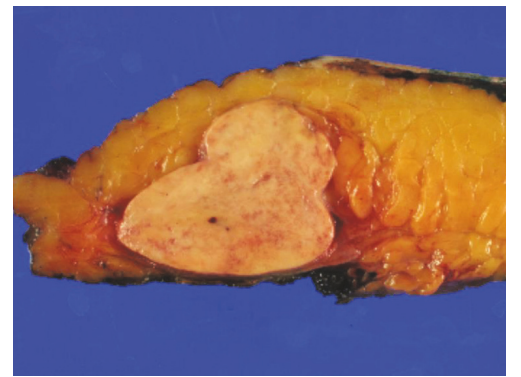

(a)

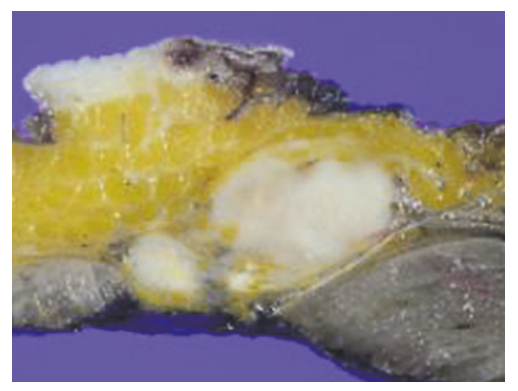

(d)

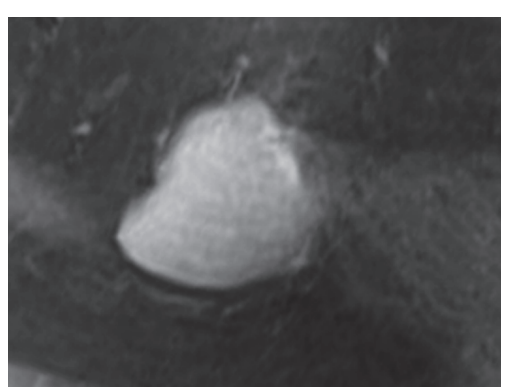

(b)

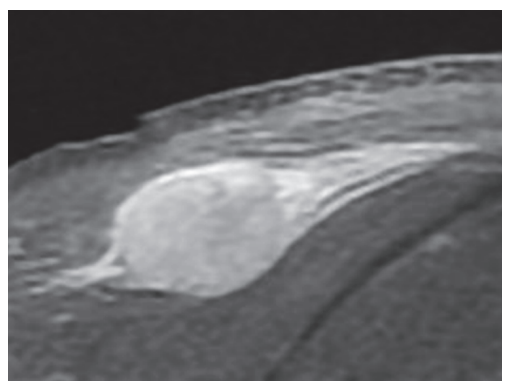

(e)

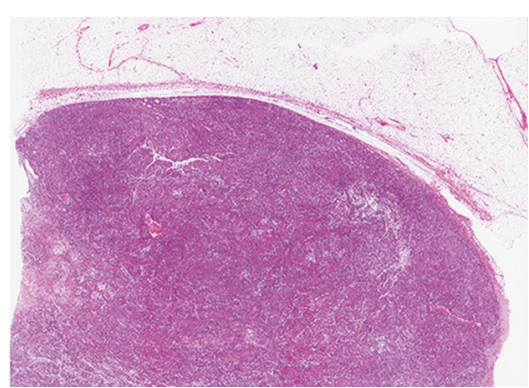

(c)

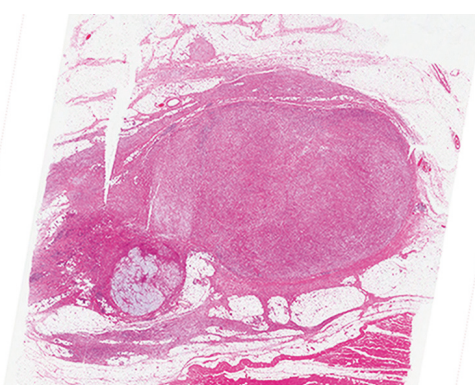

(f)

FIGURE 1: Representative sections of histologic growth pattern. Photomicrograph and MRI ((a) gross, (b) gadolinium-enhanced T1-weighted sequences with fat suppression, and (c) H\&E staining, magnification $\times 1)$ of the histological specimen of an subcutaneous synovial sarcoma with expansile growth pattern. Photomicrograph ((d) gross, (e) gadolinium-enhanced T1-weighted sequences with fat suppression, and (f) H\&E staining, magnification $\times 1$ ) of the histological specimen of a subcutaneous undifferentiated pleomorphic sarcoma with an infiltrative growth pattern.

144 patients for analysis. The institutional review board of our institute approved this study.

2.2. Assessment of Histologic Growth Pattern. The cut surface of the whole tumor was obtained from the maximum diameter and was embedded into paraffin after which $4-\mu \mathrm{m}$ sections were made. The microscopic assessment was performed on $4-\mu \mathrm{m}$ sections stained with hematoxylin-eosin. The cut surface was examined, and several representative sections were made encompassing the tumor and surrounding tissue. Microscopically the histologic growth pattern was defined as infiltrative if the penetration of the tumor cells into the surrounding tissue was observed (Figure 1(d)). Focal penetration of the tumor cells was regarded as infiltrative growth pattern. Histologic growth pattern was defined as expansile if the penetration of the tumor cells was not observed regardless of the presence of the pseudocapsule (Figure 1(a)) [10,12].

2.3. Analyses of Factors Predictive of Infiltrative Histologic Growth Pattern. Medical records were reviewed for the potential radiological and clinical factors that might be predictive of infiltrative histologic growth pattern in STS: (1) patient demographics, (2) tumor characteristics, and (3) radiological characteristics. For patient demographics, gender, age, presentation status, and history of adjuvant therapy were investigated. There were 63 women (44\%) and 81 men $(56 \%)$. The mean age at the time of STS diagnosis was 50 years (range, 5-86 years). Twenty-two patients (15\%) presented with locally recurrent tumors and 21 patients $(15 \%)$ presented after an unplanned removal of a STS. Twenty patients (14\%) had metastatic disease at the time of diagnosis of STS. STS were resected with wide margin in 125 cases (87\%), marginal margin in 18 cases (12\%), and intralesional margin in 1 case $(1 \%)$. Pathologically negative margins were achieved in 131 patients $(90 \%)$. Radiation therapy was administered in 83 patients (57\%). All patients received external beam radiation and the median dose was 60 Gy (range, 50-65 Gy). Chemotherapy was administered in 28 patients (20\%) with 10 patients $(7 \%)$ receiving preoperative chemotherapy (Table 1).

For tumor characteristics, tumor location, histologic diagnosis, histologic grade, tumor size, and tumor depth were investigated. STS were commonly located at thigh $(n=61$, $42 \%)$, lower leg $(n=19,13 \%)$, knee $(n=13,9 \%)$, buttock $(n=20,14 \%)$, shoulder $(n=11,8 \%)$, upper arm $(n=9$, $6 \%)$, forearm $(n=5,4 \%)$, and trunk wall $(n=6,4 \%)$. Most common histological diagnoses were undifferentiated pleomorphic sarcoma (UPS, $n=26,18 \%$ ), synovial sarcoma $(n=23,16 \%)$, myxofibrosarcoma $(n=22,15 \%)$, liposarcoma $(n=17,12 \%)$, and leiomyosarcoma $(n=14,10 \%)$ (Supplementary Table 1 in Supplementary Material available online at https://doi.org/10.1155/2017/5419394). Patients with welldifferentiated liposarcoma were excluded. As for histologic grading, there were 23 grade 1 (16\%), 54 grade 2 (38\%), and 67 grade $3(46 \%)$ tumors according to the Federation Nationale des Centres de Lutte Contre le Cancer (FNCLCC) classification system [13]. Mean size of the primary tumor, measured by the largest diameter on preoperative MRI, was $7.7 \mathrm{~cm}$ (range, $2.0-42.5 \mathrm{~cm}$ ). For tumor depth, tumors 
TABLE 1: Preoperative characteristics.

\begin{tabular}{|c|c|}
\hline Patient characteristics & \\
\hline Mean age $\left(y r s, S^{a}\right)$ & $50.0(19.7)$ \\
\hline \multicolumn{2}{|l|}{ Gender $(n, \%)$} \\
\hline Female & $63(44)$ \\
\hline Male & $81(56)$ \\
\hline \multicolumn{2}{|l|}{ Presentation status $(n, \%)$} \\
\hline Fresh & $101(70)$ \\
\hline Recurred & $22(15)$ \\
\hline Unplanned excision & $21(15)$ \\
\hline \multicolumn{2}{|l|}{ Radiation therapy $(n, \%)$} \\
\hline Preoperative & $1(1)$ \\
\hline Postoperative & $82(57)$ \\
\hline Not done & $61(42)$ \\
\hline Dose of radiation therapy (Gy, range) & $60(50-65)$ \\
\hline \multicolumn{2}{|l|}{ Chemotherapy $(n, \%)$} \\
\hline Preoperative & $3(2)$ \\
\hline Postoperative & $18(13)$ \\
\hline Pre- and postoperative & $7(5)$ \\
\hline Not done & $116(80)$ \\
\hline \multicolumn{2}{|l|}{ Tumor characteristics } \\
\hline Size $(\mathrm{cm}, \mathrm{SD})$ & $7.7(6.4)$ \\
\hline \multicolumn{2}{|l|}{ Depth $(n, \%)$} \\
\hline Superficial & $21(15)$ \\
\hline Deep & $123(85)$ \\
\hline \multicolumn{2}{|l|}{ Compartmental status $(n, \%)$} \\
\hline Intracompartmental & $91(63)$ \\
\hline Extracompartmental & $53(37)$ \\
\hline \multicolumn{2}{|l|}{ FNCLCC grade $(n, \%)$} \\
\hline 1 & $23(16)$ \\
\hline 2 & $54(38)$ \\
\hline 3 & $67(46)$ \\
\hline \multicolumn{2}{|l|}{ Histologic type $(n, \%)$} \\
\hline $\mathrm{UPS}^{\mathrm{b}}$ & $26(18)$ \\
\hline Synovial sarcoma & $23(16)$ \\
\hline Myxofibrosarcoma & $22(15)$ \\
\hline Liposarcoma & $17(12)$ \\
\hline Leiomyosarcoma & $14(10)$ \\
\hline Others & $42(29)$ \\
\hline \multicolumn{2}{|l|}{ MRI characteristics } \\
\hline \multicolumn{2}{|l|}{ Peritumoral edema $(n, \%)$} \\
\hline Absent & $105(73)$ \\
\hline Present & $39(27)$ \\
\hline \multicolumn{2}{|l|}{ Tumor border on MRI $(n, \%)$} \\
\hline Pushing & $57(40)$ \\
\hline Infiltrative & $87(60)$ \\
\hline
\end{tabular}

${ }^{\mathrm{a}} \mathrm{SD}$, standard deviation; ${ }^{\mathrm{b}} \mathrm{UPS}$; undifferentiated pleomorphic sarcoma.

located above the superficial fascia were defined as superficial. Twenty-one tumors (15\%) were superficial and 123 tumors were deep seated $(85 \%)$ (Table 1$)$.

For radiological characteristics, compartmental status [14], presence of peritumoral edema $[15,16]$, and the tumor border [7] were investigated using preoperative MRI scans. Spin-echo T1-weighted sequences, fast spine-echo T2weighted sequences with fat suppression, and gadoliniumenhanced T1-weighted sequences with fat suppression were available in all MRIs. Gadolinium-enhanced T1-weighted sequences were obtained in at least two orthogonal planes. MRI scans were performed with a 1.5- or 3-T system. MRI images were reviewed by two orthopaedic oncologists (J. W. $\mathrm{P}$ and $\mathrm{I} . \mathrm{H})$ and a radiologist $(\mathrm{H}$. J. Y) with expertise in musculoskeletal imaging. Compartmental status was defined as intracompartmental or extracompartmental as described by Enneking et al. [14]. There were 91 intracompartmental $(63 \%)$ and 53 extracompartmental tumors (37\%). Peritumoral edema was defined as diffuse regions of increased T2weighted signal intensity surrounding the tumor $[15,16]$. Of the 144 tumors, 39 tumors (27\%) showed peritumoral edema. Tumor border on MRI was assessed on T2-weighted and enhanced T1-weighted images. Pushing border was defined as a well-defined border without peripheral extension to the surrounding tissue (Figure 2(a)), while infiltrative border was defined as an irregular border with spicula-like extensions into the surrounding tissue (Figures 2(b) and 2(c)) [7, 1721]. Infiltrative border on MRI was identified as two different patterns: the first pattern with the tail sign, defined as a curvilinear shaped tapered thick fascial enhancement extending from the primary mass, with or without irregularity of tumor border (Figure 2(b)) [8, 17-20]; the second pattern with irregular or spiculated borders extending to surrounding tissue without the tail sign (Figure 2(c)). Of the 144 tumors, 87 tumors $(60 \%)$ had infiltrative border and $57(40 \%)$ had pushing border. Of the 87 tumors with infiltrative border, 68 tumors showed the tail sign while 19 tumors showed infiltrative border without the tail sign (Table 1).

2.4. Statistical Analyses. Continuous measures were compared using the independent-samples $t$-test and categorical variables were compared using Pearson's chi-squared test. Clinical or radiological factors which were found to have a statistically significant association with the histologic growth pattern $(p<0.05)$ were included in a multivariate logistic regression analysis, with backward selection using the likelihood ratio test, to evaluate associations linking the histologic growth pattern. Statistical analysis was performed using SPSS v.21.0 software (IBM Inc., Armonk, New York). To test the predictive accuracy of identified variables, receiveroperating characteristic (ROC) curves were constructed, and the area under the curve (AUC) with a 95\% confidence interval was calculated. Pairwise AUC comparisons were also performed between the two variables at using the nonparametric approach developed by DeLong et al. [22]. For all statistical comparisons, a $p$ value of 0.05 was considered statistically significant.

\section{Results}

3.1. Comparison of Characteristics between Patients with Infiltrative Histologic Growth Pattern and Those with Expansile Histologic Growth Pattern. Of the 144 tumors, 71 (49\%) showed histologic infiltrative growth pattern. STS with 
TABLE 2: Comparison of preoperative characteristics by histological growth pattern.

\begin{tabular}{|c|c|c|c|}
\hline & Expansile & Infiltrative & $p$ value \\
\hline Mean age (years, $\mathrm{SD}^{\mathrm{a}}$ ) & $48(20)$ & $52(20)$ & 0.14 \\
\hline Gender $(n)$ & & & 0.52 \\
\hline Female & 30 & 33 & \\
\hline Male & 43 & 38 & \\
\hline Preoperative radiation therapy $(n)$ & & & 0.49 \\
\hline Done & 0 & 1 & \\
\hline Not done & 73 & 70 & \\
\hline Preoperative chemotherapy $(n)$ & & & 0.53 \\
\hline Done & 4 & 6 & \\
\hline Not done & 69 & 65 & \\
\hline Depth $(n)$ & & & 0.03 \\
\hline Superficial & 6 & 15 & \\
\hline Deep & 67 & 56 & \\
\hline Size $(\mathrm{cm}, \mathrm{SD})$ & $7.7(5.1)$ & $7.6(7.5)$ & 0.91 \\
\hline Compartmental status $(n)$ & & & 0.52 \\
\hline Intracompartmental & 48 & 43 & \\
\hline Extracompartmental & 25 & 28 & \\
\hline Presentation status $(n)$ & & & 0.20 \\
\hline Fresh & 56 & 45 & \\
\hline Recurred & 8 & 14 & \\
\hline Unplanned excision & 9 & 12 & \\
\hline Peritumoral edema $(n)$ & & & 0.65 \\
\hline Absent & 52 & 53 & \\
\hline Present & 21 & 18 & \\
\hline Tumor border on MRI $(n)$ & & & 0.01 \\
\hline Pushing & 37 & 20 & \\
\hline Infiltrative & 36 & 51 & \\
\hline FNCLCC grade $(n)$ & & & 0.83 \\
\hline 1 & 13 & 10 & \\
\hline 2 & 27 & 27 & \\
\hline 3 & 33 & 34 & \\
\hline Surgical margin $(n)$ & & & 0.32 \\
\hline Wide & 66 & 59 & \\
\hline Marginal & 7 & 11 & \\
\hline Intralesional & 0 & 1 & \\
\hline Pathologic margin $(n)$ & & & 0.35 \\
\hline Negative & 68 & 63 & \\
\hline Positive & 5 & 8 & \\
\hline Local recurrence $(n)$ & & & 0.37 \\
\hline No & 59 & 53 & \\
\hline Yes & 14 & 18 & \\
\hline Metastasis $(n)$ & & & 0.56 \\
\hline No & 46 & 48 & \\
\hline Yes & 27 & 23 & \\
\hline
\end{tabular}

${ }^{\mathrm{a}} \mathrm{SD}$, standard deviation.

infiltrative histologic growth pattern were more likely to be superficial $(p=0.03)$ and to show infiltrative tumor border on MRI ( $p=0.01$ ) (Table 2). Among the preoperative factors analyzed, there were no significant difference found with respect to age, gender, administration of preoperative radiation therapy or chemotherapy, tumor size, compartmental status, presentation status, or presence of peritumoral edema on MRI. STS with infiltrative histologic growth pattern showed trend towards having higher chance of pathologically positive margins and local recurrence (Table 2).

3.2. Factors Associated with Infiltrative Histologic Growth Pattern. On univariate logistic regression analysis of associated factors of infiltrative histologic growth pattern in STS, superficial location $(p=0.03)$, infiltrative tumor border on MRI $(p=0.01)$, and histologic subtype other than liposarcoma ( $p=0.01)$ were significant (Table 3$)$. On multivariate analysis, infiltrative tumor border on MRI (OR $=2.48, p=0.01)$ and histological subtype other than liposarcoma $(\mathrm{OR}=4.57, p=0.02)$ remained as independent factors associated with infiltrative histologic growth pattern (Table 3).

3.3. Predictive Accuracy of Identified Factors. To test the accuracy of predicting the presence of infiltrative histologic growth pattern, we generated a predictive index based on the presence or absence of two significant factors found in the multivariate analysis. Cases with both histological subtype other than liposarcoma and infiltrative tumor border on MRI were considered to have positive predictive index. All other cases were considered to have negative predictive index.

ROC curves yielded an AUC of 0.613 (95\% CI = $0.520-0.705)$ for the tumor border on MRI, 0.575 (95\% CI = 0.481-0.668) for the histological subtype other than liposarcoma, and 0.647 (95\% CI $=0.556-0.737)$ for the predictive index. The improvement of AUC by the predictive index was significant in comparison with infiltrative tumor border on MRI alone $(p=0.010)$ (Figure 3(a)) or histological subtype other than liposarcoma alone $(p=0.028)$ (Figure $3(b))$.

\section{Discussion}

Infiltrative histologic growth pattern has been associated with poor oncologic outcome, not only for local control but also for survival in extremity STS $[7,8]$. Preoperative assessment of infiltrative histologic growth pattern would be helpful in planning treatment of extremity STS. This study examined various preoperative clinicopathologic factors and identified nonliposarcoma histology and infiltrative tumor border on MRI as independent predictors of infiltrative histologic growth pattern. To our knowledge, this is the first study to examine various preoperative factors that can predict histologic growth pattern in extremity STS.

A few things should be considered while interpreting the results of this study. First, this study was based on retrospective review of a patient cohort from a single tertiary referral hospital with relatively small number of patients. The results of this study need to be validated in external databases in a prospective setting. Second, as for the evaluation of MRI characteristics, the MRI protocols were not uniform because of the retrospective nature of the study. Third, in our routine practice, the pathologic findings according to 


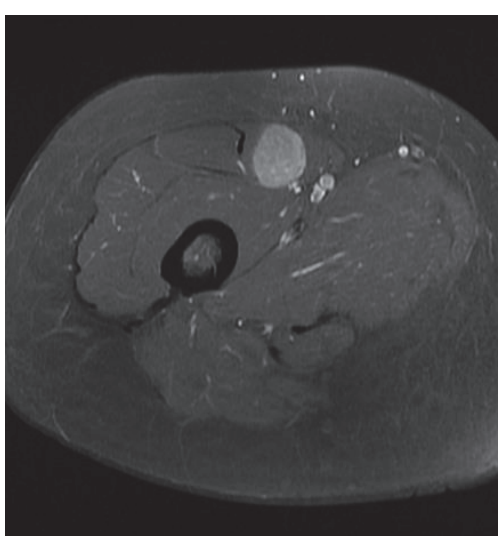

(a)

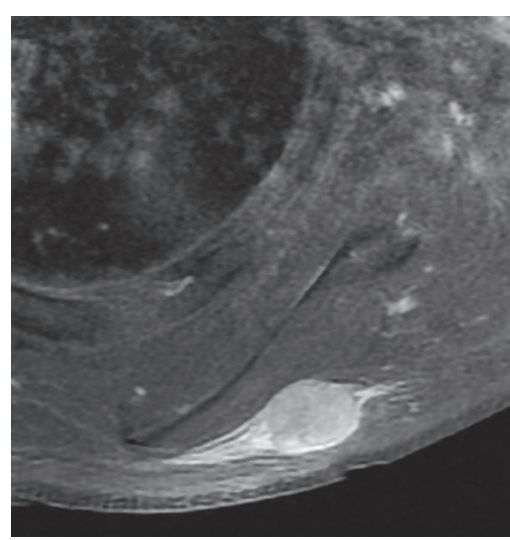

(b)

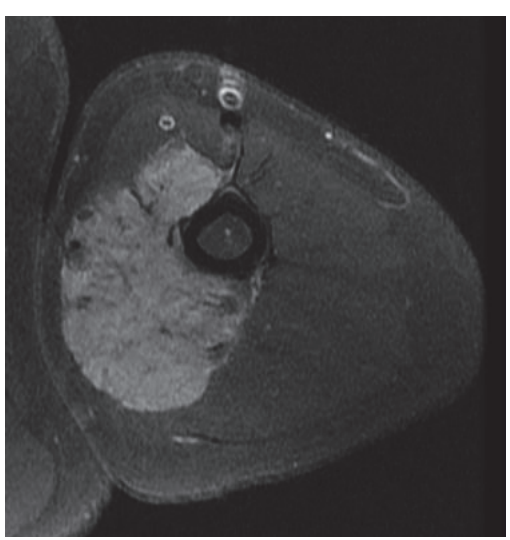

(c)

FIGURE 2: Representative images of different tumor borders on MRI. (a) T1 enhanced MRI of an intramuscular synovial sarcoma with pushing border on MRI. (b) T1 enhanced MRI of an intramuscular undifferentiated pleomorphic sarcoma with infiltrative border on MRI with the tail sign. (c) T1 enhanced MRI of a leiomyosarcoma with infiltrative border on MRI without the tail sign.

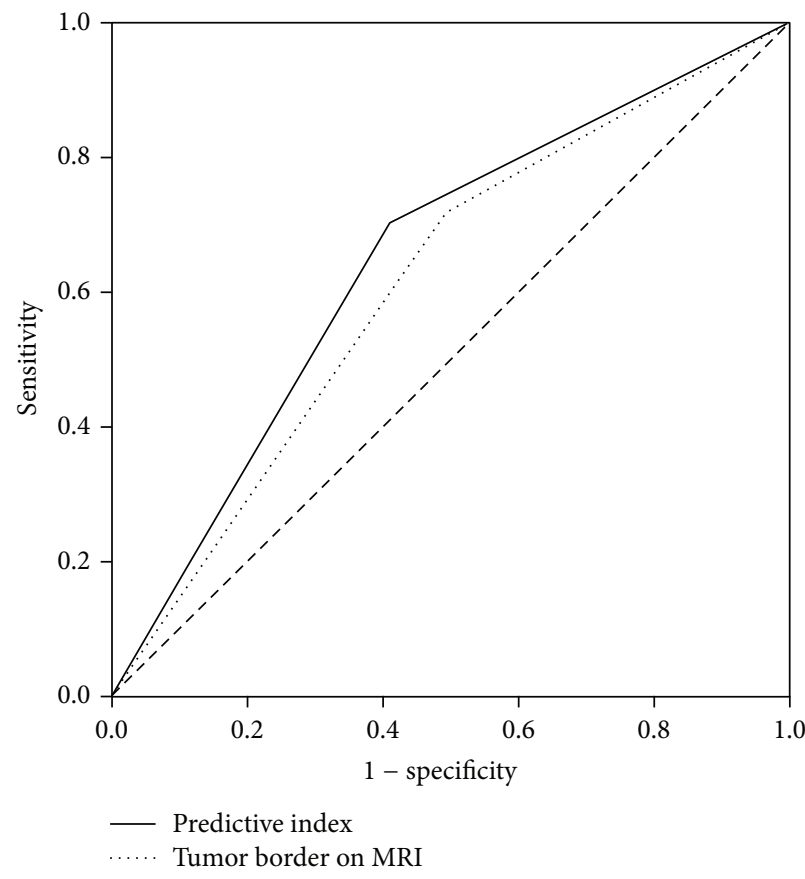

(a)

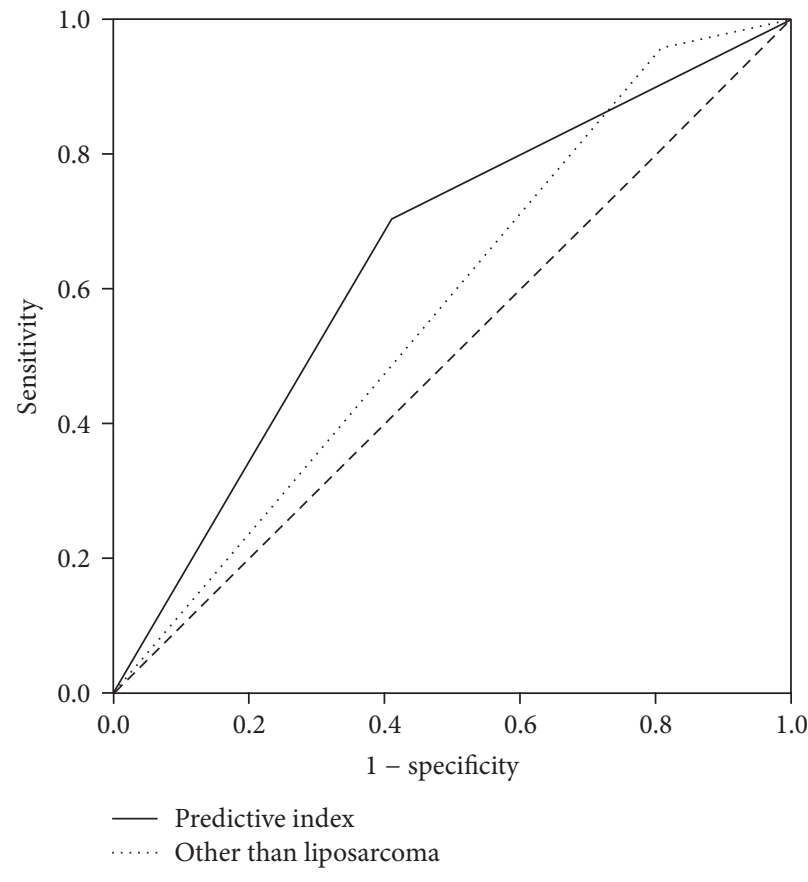

(b)

FIGURE 3: Pairwise AUC comparison of the predictive index and the tumor border on MRI alone or histological subtype other than liposarcoma alone in predicting infiltrative histologic growth pattern. (a) Pairwise area under the receiver-operating curve (AUC) comparison of the predictive index and the tumor border on MRI alone in predicting infiltrative histological growth pattern. (b) Pairwise area under the receiver-operating curve (AUC) comparison of the predictive index and histological subtype other than liposarcoma alone in predicting infiltrative histological growth pattern. AUC, area under the curve. The dashed line in both graphs represented random guess to predict $(\mathrm{AUC}=0.500)$.

the specific radiological abnormalities were not evaluated, and such radiological-pathological correlation could not be performed due to the retrospective nature of the study. Fourth, some of the factors might not be accurately assessed preoperatively, such as the histological subtype or histological grade, especially in cases where core needle biopsy is used. Fifth, the limited use of recently developed biomarkers, such as MDM2, may hamper the accurate diagnosis of histological subtype.

In this study, larger tumor size was not associated with infiltrative histological growth pattern. The authors excluded well-differentiated liposarcomas, which often present with large tumor size, despite having a less aggressive biology. However, the association between tumor size and histological 
TABLE 3: Preoperative factors associated with histological growth pattern.

\begin{tabular}{|c|c|c|c|c|c|c|}
\hline \multirow{2}{*}{ Factors } & \multicolumn{3}{|c|}{ Univariate } & \multicolumn{3}{|c|}{ Multivariate } \\
\hline & $\mathrm{OR}^{\mathrm{a}}$ & $95 \% \mathrm{CI}^{\mathrm{b}}$ & $p$ value & OR & $95 \% \mathrm{CI}$ & $p$ value \\
\hline Age & 1.01 & $1.00-1.03$ & 0.14 & & & \\
\hline Gender & & & 0.80 & & & \\
\hline Female & 1.00 & & & & & \\
\hline Male & 0.80 & $0.41-1.55$ & & & & \\
\hline Preoperative radiation therapy & & & 1.00 & & & \\
\hline Done & 1.00 & & & & & \\
\hline Not done & 1.00 & & & & & \\
\hline Preoperative chemotherapy & & & 0.49 & & & \\
\hline Done & 1.59 & $0.43-5.90$ & & & & \\
\hline Not done & 1.00 & & & & & \\
\hline Depth & & & $0.03^{c}$ & & & \\
\hline Superficial & 3.00 & $1.09-8.22$ & & & & \\
\hline Deep & 1.00 & & & & & \\
\hline Size & 1.00 & $0.95-1.05$ & 0.91 & & & \\
\hline Compartmental status & & & 0.51 & & & \\
\hline Intracompartmental & 1.00 & & & & & \\
\hline Extracompartmental & 1.25 & $0.63-2.46$ & & & & \\
\hline Previous treatment & & & 0.20 & & & \\
\hline Fresh & 1.00 & & & & & \\
\hline Recurred & 2.18 & $0.84-5.65$ & & & & \\
\hline Unplanned excision & 1.66 & $0.64-4.29$ & & & & \\
\hline Peritumoral edema & & & 0.65 & & & \\
\hline Absent & 1.00 & & & & & \\
\hline Present & 0.84 & $0.40-1.76$ & & & & \\
\hline Tumor border on MRI & & & $0.01^{\mathrm{c}}$ & & & $0.01^{\mathrm{c}}$ \\
\hline Pushing & 1.00 & & & 1.00 & & \\
\hline Infiltrative & 2.62 & $1.31-5.23$ & & 2.48 & $1.21-5.08$ & \\
\hline FNCLCC grade & & & 0.83 & & & \\
\hline 1 & 1.00 & & & & & \\
\hline 2 & 1.30 & $0.49-3.47$ & & & & \\
\hline 3 & 1.34 & $0.52-3.48$ & & & & \\
\hline Liposarcoma & & & $0.01^{\mathrm{c}}$ & & & $0.02^{\mathrm{c}}$ \\
\hline Liposarcoma & 1.00 & & & 1.00 & & \\
\hline Others & 5.38 & $1.47-19.63$ & & 4.57 & $1.22-17.05$ & \\
\hline
\end{tabular}

${ }^{\mathrm{a}} \mathrm{OR}$, odds ratio; ${ }^{\mathrm{b}} \mathrm{CI}$, confidence interval; ${ }^{\mathrm{c}}$ statistically significant.

growth pattern remained insignificant even after the exclusion of well-differentiated liposarcomas.

Histological subtypes of STS seems to be associated with histologic growth pattern. Majority of STS subtypes showed predominantly infiltrative histologic growth pattern; UPS $(62 \%, 16 / 26)$, myxofibrosarcoma $(59 \%, 13 / 22)$, and synovial sarcoma $(57 \%, 13 / 23)$. However, predominantly expansile histologic growth pattern was observed in MPNST $(87 \%, 7 / 8)$ and liposarcoma $(82 \%, 14 / 17)$. As current treatment strategies for STS are increasingly adapted to a specific histological subtype, predictive factors for specific subtypes of STS will be needed.

The value of tumor border on MRI in predicting histologic growth pattern seem to differ among histological subtypes of STS. The association of infiltrative tumor border on MRI, most notably the tail sign, with infiltrative histologic growth pattern has been well-documented in UPS and myxofibrosarcoma [7, 17-20, 23]. Indeed, tumor border on MRI predicted infiltrative histologic growth pattern in UPS (AUC $=0.619,95 \% \mathrm{CI}=0.385-0.852$ ) and myxofibrosarcoma (AUC $=0.649,95 \% \mathrm{CI}=0.391-0.907)$ with moderate accuracy in this study. However, for synovial sarcoma, ROC curve yielded an AUC of 0.519 (95\% CI = 0.276-0.762). These data suggest that the predictive value of tumor border on MRI in assessing histologic growth pattern differs among histologic subtypes of STS.

Histologic growth patterns may be affected by the tumor environment. Tumors located in the superficial location, where no fascial boundaries exist, had more tumors with infiltrative histologic growth pattern than tumors located 
in the deep location in the univariate analysis. Moreover, tumors presenting with previous unplanned surgery or local recurrence, in which the normal anatomical boundaries are violated, had more tumors with infiltrative histologic growth pattern than tumors presenting without any previous surgeries. Taken together, histologic growth pattern seems to be affected not only by the tumor biology but also by the tumor environment.

The predictive accuracy of the predictive index generated in this study was at best moderate with an AUC value of 0.647. Better predictive factors, such as genomic markers, are needed to improve predicting the histologic growth pattern in extremity STS. However, the results of this study can be implemented easily in the clinical practice and may provide a backbone for newly identified predictive factors of histologic growth pattern in extremity STS.

\section{Conclusions}

In conclusion, our data suggests that liposarcoma histology and tumor border on MRI can predict histologic growth pattern in extremity STS. If an extremity STS of nonliposarcoma histology shows infiltrative tumor border on MRI, infiltrative histologic growth pattern can be expected.

\section{Additional Points}

Our data suggest that patients with histological diagnosis other than liposarcoma and the presence of infiltrative border on MRI can predict infiltrative histologic growth pattern in extremity soft tissue sarcoma.

\section{Disclosure}

This paper was presented as a poster at The 19th International Society of Limb Salvage General Meeting.

\section{Conflicts of Interest}

The authors have declared no conflicts of interest or financial support.

\section{References}

[1] N. J. Espat and J. J. Lewis, “The biological significance of failure at the primary site on ultimate survival in soft tissue sarcoma," Seminars in Radiation Oncology, vol. 9, no. 4, pp. 369-377, 1999.

[2] C. S. Trovik, "Local recurrence of soft tissue sarcoma. a Scandinavian sarcoma group project," Acta Orthopaedica Scandinavica, vol. 72, no. 300, pp. 1-31, 2001.

[3] J. Weitz, C. R. Antonescu, and M. F. Brennan, "Localized extremity soft tissue sarcoma: improved knowledge with unchanged survival over time," Journal of Clinical Oncology, vol. 21, no. 14, pp. 2719-2725, 2003.

[4] G. K. Zagars, M. T. Ballo, P. W. T. Pisters et al., "Prognostic factors for patients with localized soft-tissue sarcoma treated with conservation surgery and radiation therapy: an analysis of 1225 patients," Cancer, vol. 97, no. 10, pp. 2530-2543, 2003.
[5] C. D. Fletcher, "The evolving classification of soft tissue tumours-an update based on the new 2013 WHO classification," Histopathology, vol. 64, pp. 2-11, 2014.

[6] S. Tsukushi, Y. Nishida, J. Wasa, H. Urakawa, and N. Ishiguro, "Clinicopathological assessment of T1 soft tissue sarcomas," Archives of Orthopaedic and Trauma Surgery, vol. 131, no. 5, pp. 695-699, 2011.

[7] J. Fernebro, M. Wiklund, K. Jonsson et al., "Focus on the tumour periphery in MRI evaluation of soft tissue sarcoma: Infiltrative growth signifies poor prognosis," Sarcoma, vol. 2006, Article ID 21251, 2006.

[8] S. Iwata, T. Yonemoto, A. Araki et al., "Impact of infiltrative growth on the outcome of patients with undifferentiated pleomorphic sarcoma and myxofibrosarcoma," Journal of Surgical Oncology, vol. 110, no. 6, pp. 707-711, 2014.

[9] M. W. Manoso, J. Pratt, J. H. Healey, P. J. Boland, and E. A. Athanasian, "Infiltrative MRI pattern and incomplete initial surgery compromise local control of myxofibrosarcoma," Clinical Orthopaedics and Related Research, vol. 450, pp. 89-94, 2006.

[10] J. Engellau, P.-O. Bendahl, A. Persson et al., "Improved prognostication in soft tissue sarcoma: Independent information from vascular invasion, necrosis, growth pattern, and immunostaining using whole-tumor sections and tissue microarrays," Human Pathology, vol. 36, no. 9, pp. 994-1002, 2005.

[11] K. R. Gundle, S. Gupta, L. Kafchinski et al., "An Analysis of Tumor- and Surgery-Related Factors that Contribute to Inadvertent Positive Margins Following Soft Tissue Sarcoma Resection," Annals of Surgical Oncology, vol. 24, no. 8, pp. 21372144, 2017.

[12] B. Skytting, J. M. Meis-Kindblom, O. Larsson et al., "Synovial sarcoma - Identification of favorable and unfavorable histologic types: A Scandinavian sarcoma group study of 104 cases," Acta Orthopaedica Scandinavica, vol. 70, no. 6, pp. 543-554, 2009.

[13] J. M. Coindre, "Grading of soft tissue sarcomas: review and update," Archives of Pathology \& Laboratory Medicine, vol. 130, no. 10, pp. 1448-1453, 2006.

[14] W. F. Enneking, S. S. Spanier, and M. M. Malawer, "The effect of the anatomic setting on the results of surgical procedures for soft parts sarcoma of the thigh," Cancer, vol. 47, no. 5, pp. 1005$1022,1981$.

[15] L. M. White, J. S. Wunder, R. S. Bell et al., "Histologic assessment of peritumoral edema in soft tissue sarcoma," International Journal of Radiation Oncology Biology Physics, vol. 61, no. 5, pp. 1439-1445, 2005.

[16] S. Kang, H. J. Yoo, H.-S. Kim, and I. Han, "Soft tissue sarcoma misdiagnosed as benign peripheral neurogenic tumor," Journal of Orthopaedic Science, vol. 20, no. 1, pp. 180-185, 2015.

[17] M. Kaya, T. Wada, S. Nagoya et al., "MRI and histological evaluation of the infiltrative growth pattern of myxofibrosarcoma," Skeletal Radiology, vol. 37, no. 12, pp. 1085-1090, 2008.

[18] B. Waters, D. M. Panicek, R. A. Lefkowitz et al., "Low-grade myxofibrosarcoma: CT and MRI patterns in recurrent disease," American Journal of Roentgenology, vol. 188, no. 2, pp. W193W198, 2007.

[19] R. A. Lefkowitz, J. Landa, S. Hwang et al., "Myxofibrosarcoma: Prevalence and diagnostic value of the "tail sign" on magnetic resonance imaging," Skeletal Radiology, vol. 42, no. 6, pp. 809818, 2013.

[20] H. J. Yoo, S. H. Hong, Y. Kang et al., "MR imaging of myxofibrosarcoma and undifferentiated sarcoma with emphasis on tail sign; Diagnostic and prognostic value," European Radiology, vol. 24, no. 8, pp. 1749-1757, 2014. 
[21] E. A. Walker, J. M. Petscavage, P. L. Brian, C. I. Logie, K. M. Montini, and M. D. Murphey, "Imaging features of superficial and deep fibromatoses in the adult population," Sarcoma, vol. 2012, Article ID 215810, 2012.

[22] E. R. DeLong, D. M. DeLong, and D. L. Clarke-Pearson, "Comparing the areas under two or more correlated receiver operating characteristic curves: a nonparametric approach," Biometrics, vol. 44, no. 3, pp. 837-845, 1988.

[23] G. Riouallon, F. Larousserie, E. Pluot, and P. Anract, "Superficial myxofibrosarcoma: Assessment of recurrence risk according to the surgical margin following resection. A series of 21 patients," Orthopaedics and Traumatology: Surgery and Research, vol. 99, no. 4, pp. 473-477, 2013. 


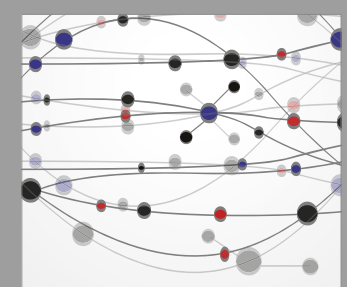

The Scientific World Journal
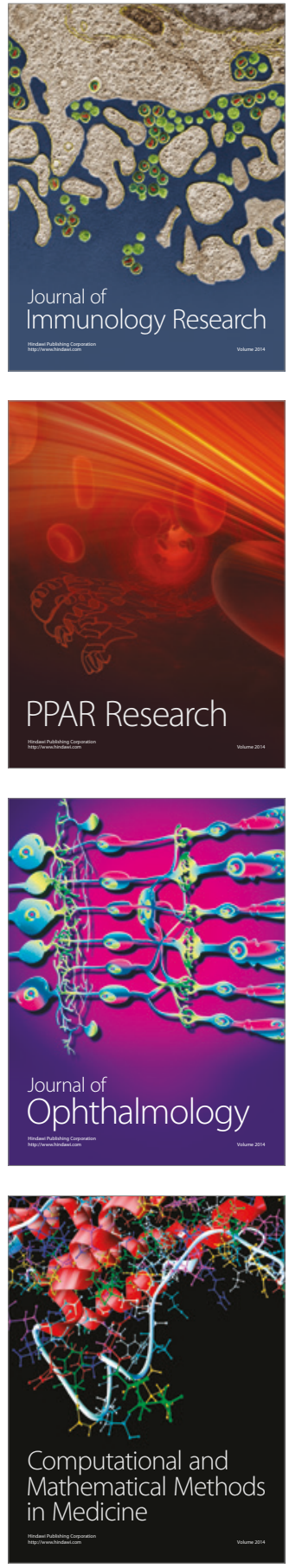

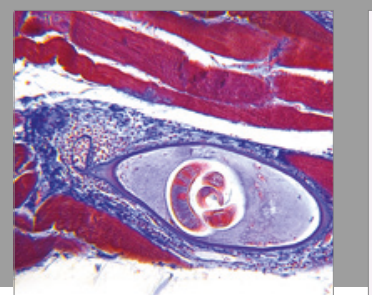

Gastroenterology Research and Practice
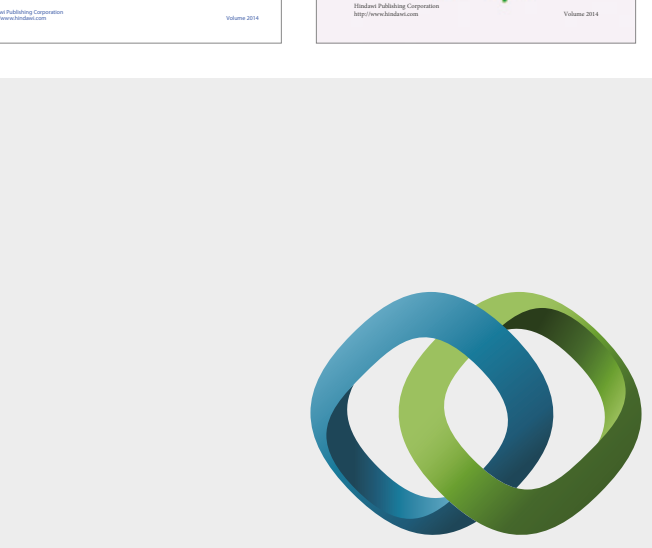

\section{Hindawi}

Submit your manuscripts at

https://www.hindawi.com
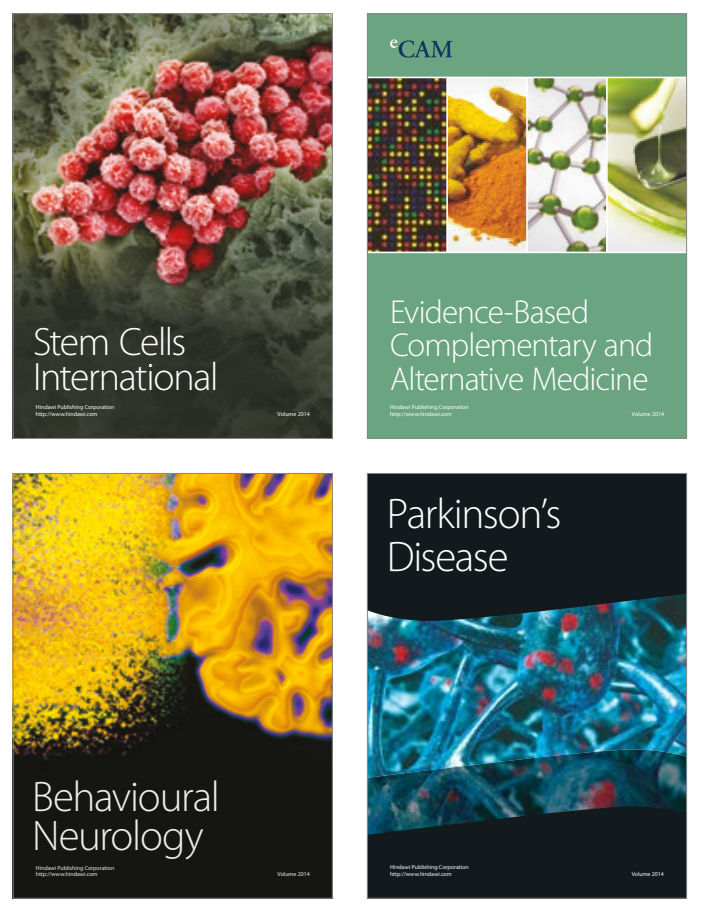
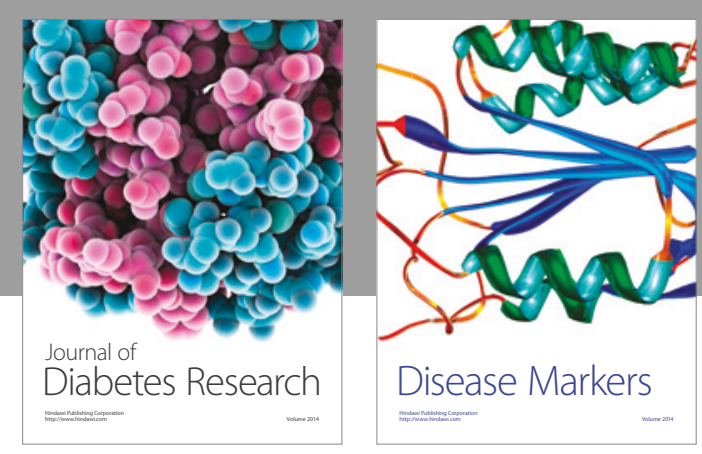

Disease Markers
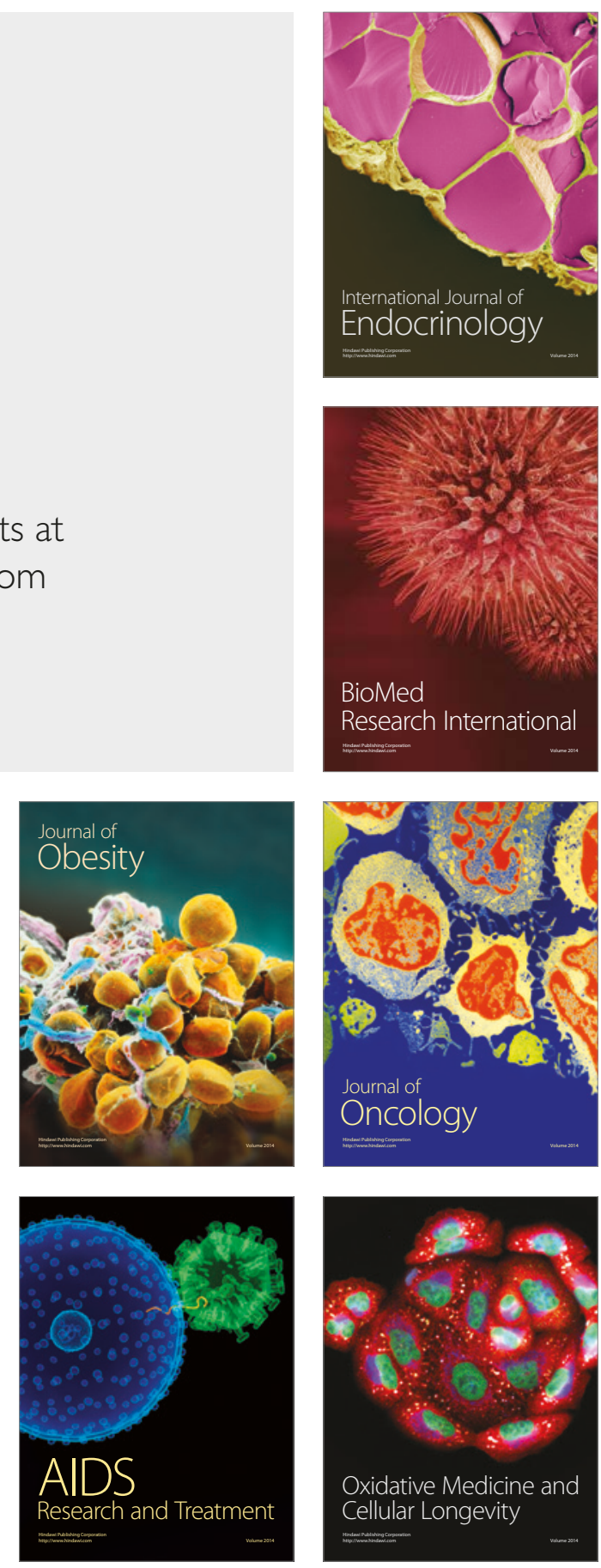